\title{
Inversion of the high-frequency source radiation of M6.8 Avachinsky Gulf, Kamchatka, earthquake using empirical and theoretical envelope Green functions
}

\author{
Anatoly G. Petukhin ${ }^{1}$, Hisashi Nakahara ${ }^{2}$, and Alexander A. Gusev ${ }^{3}$ \\ ${ }^{1}$ Geo-Research Institute, 4-3-2 Itachibori, Nishi-ku, Osaka 550-0012, Japan \\ ${ }^{2}$ Graduate School of Science, Tohoku University, Sendai, Japan \\ ${ }^{3}$ Institute of Volcanic Geology and Geochemestry, Russian Academy of Sciences, Petropavlovsk-Kamchatsky, Russia
}

(Received May 24, 2004; Revised July 12, 2004; Accepted July 12, 2004)

\begin{abstract}
We study the generation of high-frequency S-wave energy from its source of the 1992 Avachinsky Gulf, Kamchatka, earthquake ( $M_{W}=6.8$ ) by means of the envelope inversion method of Nakahara et al. (1998). Two versions of this technique were employed, the original version and the modified version. In the modified version, to construct the "envelope Green function" we use empirical envelope shapes, constructed by averaging data of small earthquakes from the same area, calibrated to the total energy of the theoretical envelopes. The envelope inversion procedure was applied to six mainshock records, producing estimates of spatial distribution of energy radiation in frequency bands: $1-2,2-4,4-8$ and $8-16 \mathrm{~Hz}$. In both inversions, the distribution of energy radiation shows a similar pattern: 75-90 percent of the energy was radiated soon after nucleation. Afterwards, the rupture propagated mostly in a westward direction. Similarity of both results demonstrates the robustness of the envelope inversion method. However the version of the inversion procedure that uses empirical envelopes yields more plausible characteristics of the rupture process.
\end{abstract}

Key words: Envelope source inversion, high-frequency source radiation, high-frequency scattering, Kamchatka.

\section{Introduction}

Envelope inversion is an efficient method to study the distribution of high-frequency (HF) seismic energy generation in an earthquake source. Iida and Hakuno (1984), using accelerogram data, were able to reconstruct the spacetime history of HF energy generation from the sources of the 1968, M7.9 Tokachi-oki, Japan, and 1978, M7.6, off Miyagi, Japan, earthquakes. For deconvolution, they used the aftershock envelope as an envelope Green function (EnvGF). This approach was further developed by Gusev and Pavlov (1991) and Kakehi et al. (1996). For not uncommon cases when usable records of fore- or aftershocks are absent, Nakahara et al. (1998), introduced an alternative approach based on the theoretical EnvGF that was calculated using the multiple isotropic scattering model of Sato et al. (1997). Using this method, several large earthquakes were successfully inverted (Nakahara et al., 1998; Nakahara et al., 1999; Nakahara and Watanabe, 2000; Nakahara, 2004). Although this approach generally produces quite an acceptable fit, at larger distances the theoretical model cannot describe well the initial part of the observed envelope shapes. The cause is the fact that the initial part of the theoretical envelopes is formed by low-angle scattering that cannot be realistically predicted by the isotropic scattering model (whereas, the later parts, related to large-angle scattering, are predicted rather well).

In the present paper, we propose a remedy to this problem

Copy right(C) The Society of Geomagnetism and Earth, Planetary and Space Sciences (SGEPSS); The Seismological Society of Japan; The Volcanological Society of Japan; The Geodetic Society of Japan; The Japanese Society for Planetary Sciences; TERRAPUB in the form of hybrid, semi-empirical envelopes. To determine the Hybrid EnvGF, we use an empirical envelope, but not that of an aftershock. Instead, we use the average envelope shape of small earthquakes generally recorded in the same area as the large earthquake in question. However, to account for the source radiation pattern and also to determine absolute amplitude levels, we calibrate these envelope shapes using the results of theoretical calculations based on the multiple isotropic scattering model.

Using new Hybrid EnvGF's, we apply the inversion method of Nakahara et al. (1998) to strong-motion records of the 1992 Avachinsky Gulf, Kamchatka, earthquake $\left(M_{W}=\right.$ 6.8 ), for the following set of frequency bands: $1-2,2-4,4-8$ and $8-16 \mathrm{~Hz}$. In parallel, to compare results, the same data were inverted using the earlier approach with purely Theoretical EnvGF's. In our study, we extensively use the results of Petukhin and Gusev (2003) who have already determined distance-dependent families of average empirical envelopes for the same area of the eastern coast of Kamchatka, independently for each of the mentioned frequency bands (1-2, $2-4,4-8$ and $8-16 \mathrm{~Hz}$ ) using broadband digital records of small earthquakes.

\section{Method of Inversion}

We use the inversion method of Nakahara et al. (1998), where the unknowns are the values of seismic energy radiation from a number of subfaults, and also values of relative site amplification factors for the observing stations. Figure 1 illustrates the underlying model for a record envelope formation. We assume that HF waves, radiated by different sub- 


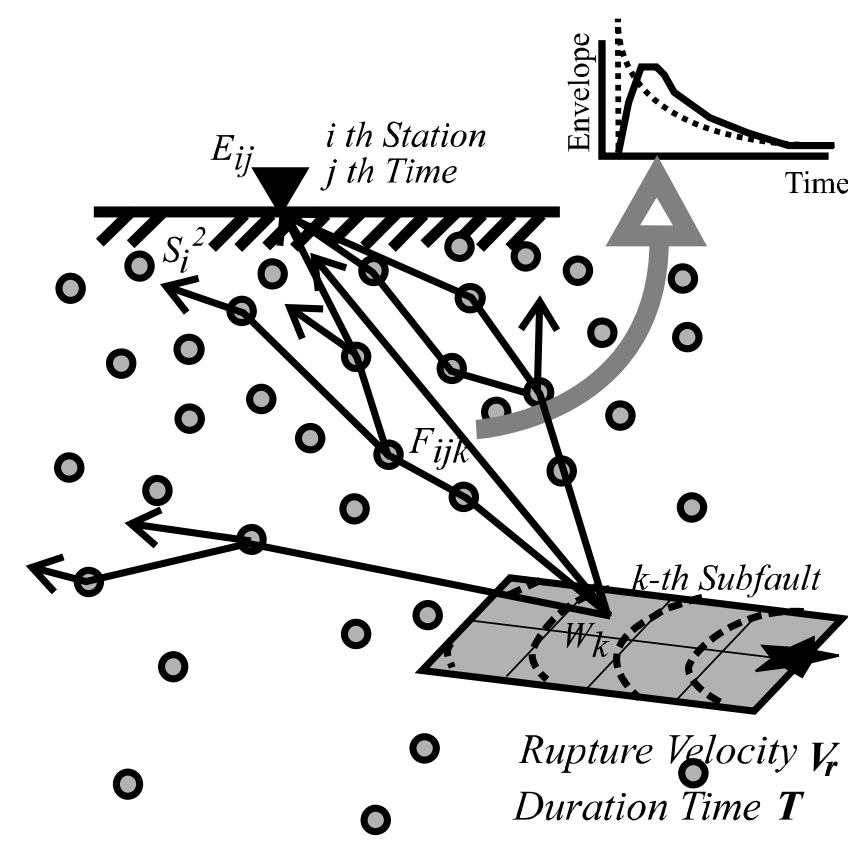

Fig. 1. A cartoon of envelope formation. Seismic energy is radiated from a double couple source located at the center of the $k$ th subfault, it is scattered mostly ahead (non-isotropic scattering, mostly due to diffraction), amplified under the $i$ th station (triangle) and reaches the station at the $j$ th time bin. Circles symbolize medium heterogeneities (scatters) distributed randomly in the space. The diagram in the upper right corner illustrates the difference between typical envelope shapes for isotropic (dashed line) and non-isotropic forward-enhanced (solid line) scattering models.

faults add up at a station in the incoherent manner, so that at any moment in time, the total energy density at a station is the sum of the energy densities produced by each subfault, with the appropriate radiation pattern and appropriate delay. We now consider a particular frequency band. Let us write down the discrete time sequence representing the EnvGF for a combination of $k$ th subfault and $i$ th station, as $e_{i k j}$, and the time history of the same subfault as $s_{k j}$, where the subscript $j$ represents time. Denote the convolution of $e_{i k j}$ and $s_{k j}$ along the time axis as $F_{i k j}$. The contribution of the $k$ th subfault to the observed envelope $E_{i j}$ at the $i$ th station within the $j$ th time step can be considered as the product of three factors: $F_{i k j}$ (known), $W_{k}$-energy radiation from the $k$ th subfault (unknown), and $S_{i}$ - site amplification factor for the $i$ th station (another unknown). Thus:

$$
E_{i j}=\sum_{k} W_{k} \cdot F_{i j k} \cdot S_{i}^{2}+\varepsilon_{i j}
$$

where $\varepsilon_{i} j$ is the random error. The unknowns $W_{k}$ and $S_{i}$ were estimated iteratively by minimizing the misfit function:

$$
\text { Misfit }=\sum_{i, j} p_{i}\left|E_{i j}-\sum_{k} S_{i}^{2} F_{i j k} W_{k}\right|^{2} \rightarrow \text { Min. }
$$

where $p_{i}=\left[\max _{j}\left(E_{i j}\right)\right]^{-2}$ is a weight coefficient introduced to balance the contributions of the various stations with broadly varying absolute amplitudes. In the described procedure, we also assume that the time history of a subfault can be described by a boxcar of duration $T$, delayed, with respect to the nucleation time, by rupture propagation at a

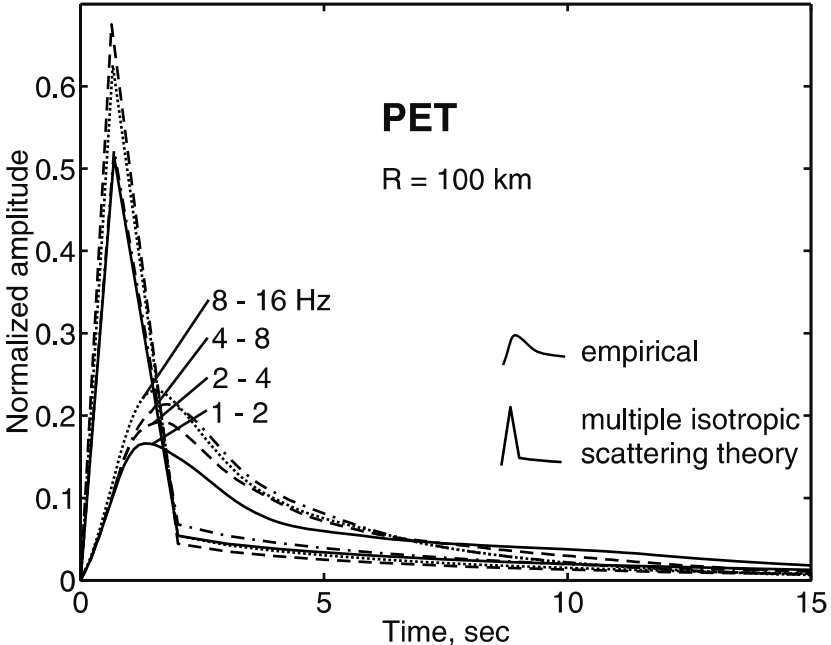

Fig. 2. Examples of theoretical and average empirical envelopes for the same distance. Total seismic energy is identical for each of the envelopes. For the "theoretical" case, where a delta-like component is present, artificial smoothing is performed assuming a sampling interval equal to 1 sec.

Table 1. Parameters of scattering and intrinsic loss used in the calculation of the theoretical envelopes (after Abubakirov and Gusev, 1990).

\begin{tabular}{ccc}
\hline $\begin{array}{c}\text { Frequency band } \\
(\mathrm{Hz})\end{array}$ & $\begin{array}{c}\text { Scattering coeff. } \\
g\left(\mathrm{~km}^{-1}\right)\end{array}$ & $\begin{array}{c}\text { Intrinsic } Q \text {-value } \\
Q_{i}\end{array}$ \\
\hline $1-2$ & 0.0077 & 195 \\
$2-4$ & 0.0048 & 304 \\
$4-8$ & 0.0065 & 488 \\
$8-16$ & 0.0100 & 840 \\
\hline
\end{tabular}

constant velocity $V_{R}$. The entire procedure was repeated for a set of $V_{R}-T$ pairs, and the optimal pair of values was selected by a grid search.

The problem (1) is non-unique: there is a trade-off between unknowns $W_{k}$ and $S_{i}$. To remove this trade-off, one of the site amplification factors $S_{i}$, corresponding to a certain reference station with hard rock geology, was fixed to be equal to 2 for the free-surface station.

\section{Envelope Green Functions}

The original inversion method of Nakahara et al. (1998) uses theoretical envelope Green functions after Sato et al. (1997), obtained following the multiple isotropic scattering model for $\mathrm{S}$ waves, further denoted as Case T. As an alternative, one could apply average empirical envelopes after Petukhin and Gusev (2003), denoted Case E. Let us consider the main features for these two alternative cases, and mark $(+)$ realistic and acceptable features, $(-)$ features that needs correction/elimination. (1) The shape of the pulse of direct waves: (T) delta-like, distance independent, frequency independent (-); (E) realistic frequency-dependent pulse shape with duration proportional to distance, the shape is consistent with the model of forward scattering of the body-wave pulse in the medium with a power-law inhomogeneity spectrum (+). (2) The source radiation pattern for S-waves: (T) accounted for $(+)$; (E) ignored, isotropic pattern is assumed instead (-). See Fig. 2 for actual envelope shapes of the two models. 
Table 2. Parameters of the 1992 Avachinsky Gulf earthquake.

\begin{tabular}{|c|c|c|c|c|c|c|c|c|}
\hline Date & Time & Lat. $^{\circ} \mathrm{N}$ & Lon. ${ }^{\circ} \mathrm{E}$ & Depth $[\mathrm{km}]$ & $M_{W}$ & strike & dip & rake \\
\hline March 2, 1992 & $12 \mathrm{~h} 29 \mathrm{~m} 38 \mathrm{~s}$ & 52.76 & 160.20 & 20 & 6.8 & $213^{\circ}$ & $28^{\circ}$ & $84^{\circ}$ \\
\hline
\end{tabular}

Table 3. Parameters of rupture estimated by the grid search method. The results obtained with hybrid EnvGF and theoretical EnvGF are given before and after the slash, respectively.

\begin{tabular}{ccc}
\hline $\begin{array}{c}\text { Frequency band } \\
f[\mathrm{~Hz}]\end{array}$ & $\begin{array}{c}\text { Rupture velocity } \\
V_{R}[\mathrm{~km} / \mathrm{s}]\end{array}$ & $\begin{array}{c}\text { Subfault duration } \\
T[\mathrm{~s}]\end{array}$ \\
\hline $1-2$ & $3.0 / 3.0$ & $0.2 / 4$ \\
$2-4$ & $3.0 / 3.0$ & $0.2 / 4$ \\
$4-8$ & $3.2 / 3.5$ & $0.2 / 2$ \\
$8-16$ & $2.5 / 3.5$ & $0.2 / 2$ \\
\hline
\end{tabular}

To combine the positive features of the two models, we created a new hybrid EnvGF model that uses the multiple isotropic scattering model to calculate total seismic energy and to account for the source radiation pattern, whereas to describe the shape of the time history, we used empirically determined shapes, i.e.,

$$
e^{(h)}(t)=\left(\frac{\int e^{(t)}(t) \mathrm{d} t}{\int e^{(a)}(t) \mathrm{d} t}\right) e^{(a)}(t)
$$

where the superscripts $t, a$ and $h$ denote theoretical, average empirical (uncalibrated) and hybrid Green functions, respectively; $e(t)$ - envelope Green function.

In the calculation of theoretical envelopes we used scattering and intrinsic loss parameters for the same area, after Abubakirov and Gusev (1990) and Petukhin et al. (1999), see Table 1. Distance-dependent families of average empirical envelopes (Fig. 2, after Petukhin and Gusev (2003)) were constructed using small earthquakes data in the same area and of a similar depth, 20-40 km. The main feature of these families is the linear increase of the width parameter (rms duration) with distance. This empirical result is in significant contradiction with the approximately quadratic increase of the duration of the forward-scattered envelope with distance (Saito et al., 2002) that is expected theoretically for body waves in a uniformly scattering medium. The contradiction seems to be caused by the fast decrease of the scattering parameters with depth.

\section{Station Distribution and Fault Geometry}

The 1992 Avachinsky Gulf earthquake (Table 2) triggered six stations of the Kamchatka Strong Motion Network, with epicentral distances from 102 to 214 km (see Fig. 3). The preferred fault plane (the least steep, "subduction" one) was selected from the Harvard CMT best double couple solution on the basis of the distribution of aftershocks (Zobin et al., 1997), see Fig. 3. It was divided into five identical subfaults of $10 \times 10 \mathrm{~km}$ each. Three central subfaults, stretching longitudinally, cover the aftershock area; two more subfaults were added at both the eastern and western ends. The hypocenter or nucleation point of the rupture is marked by a star in Fig. 3 within the subfault No. 2. We selected the fault geometry based on available aftershock data and CMT solution,

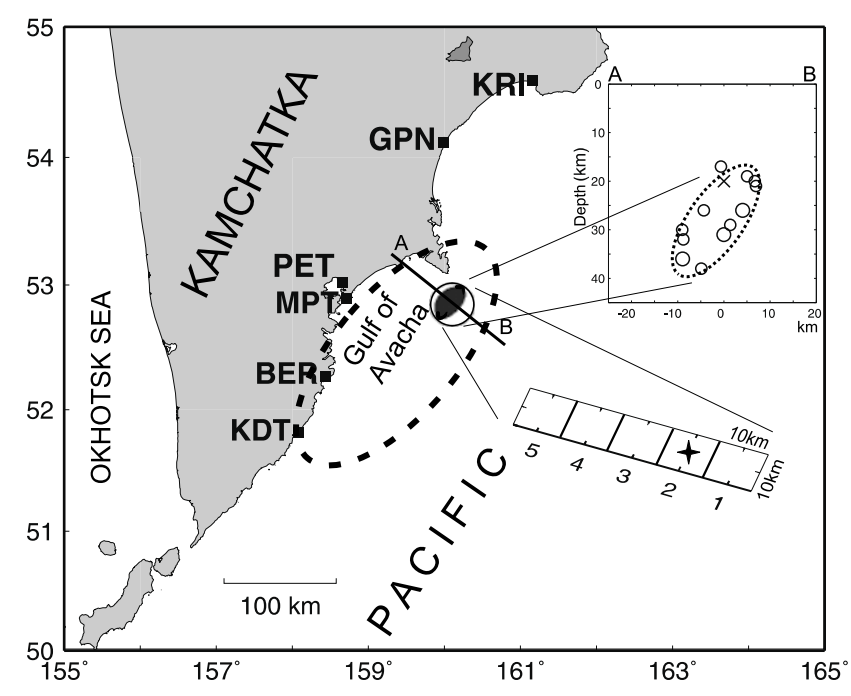

Fig. 3. Schematic map depicting strong-motion stations used in the analysis (squares), earthquake source (beach-ball), distribution of the first-day aftershocks along line A-B and assumed fault-subfault geometry. Dashed line bounds the epicentral area of small earthquakes used to construct average empirical envelopes. Star symbol in subfault No. 2 denotes the rupture nucleation point.
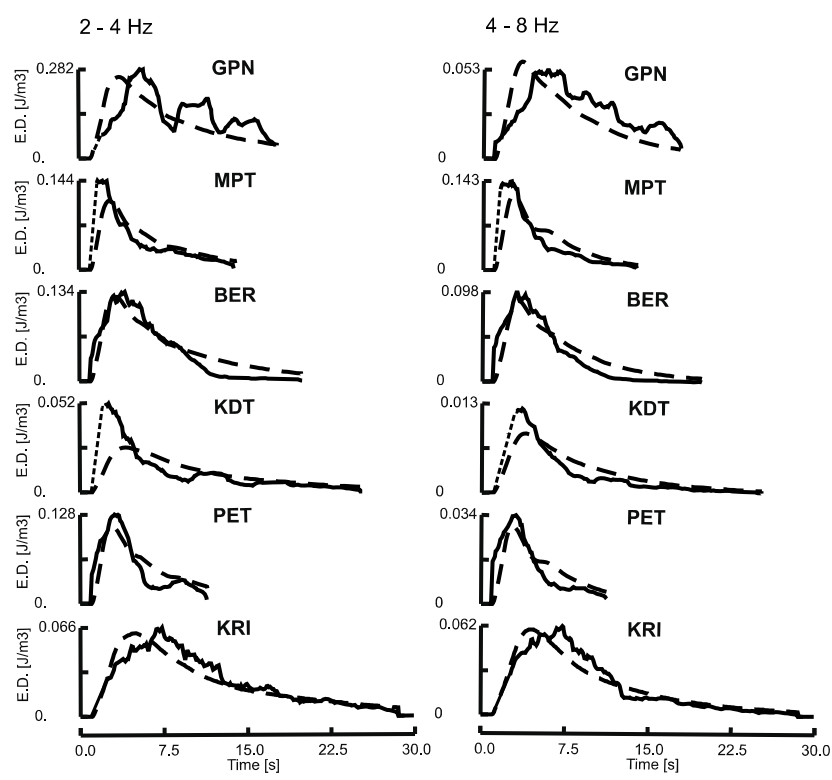

Fig. 4. Examples of fit between observed (solid line) and synthetic (dashed line) envelopes.

even if it looks unusual: the fault elongates in the dipping direction. The amount of details in the expected result for this off-shore earthquake agrees with the amount and resolution capabilities of the available data. For determination of site factors, the station PET, located on a hard rock massif, was selected as the point of reference and its site effect was assumed to be constant within the studied frequency range. 


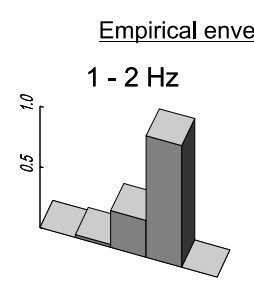

$4-8 \mathrm{~Hz}$

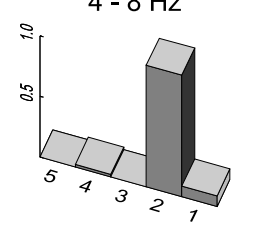

$2-4 \mathrm{~Hz}$

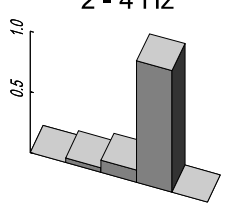

$8-16 \mathrm{~Hz}$

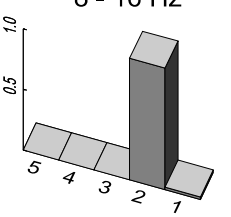

Theoretical envelope inversion
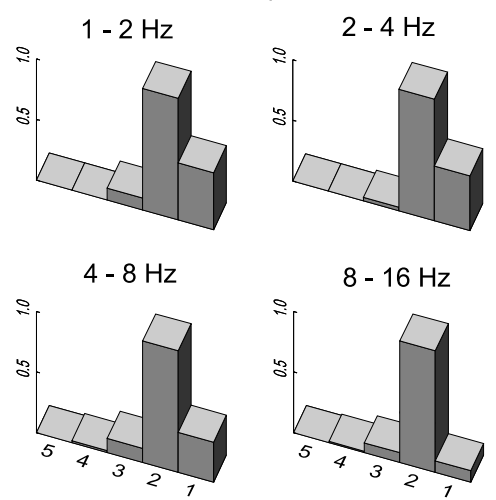

$8-16 \mathrm{~Hz}$

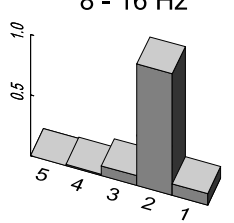

Fig. 5. Spatial distribution of high-frequency energy generation over the fault plane. Amplitudes on each diagram are normalized to the maximum.

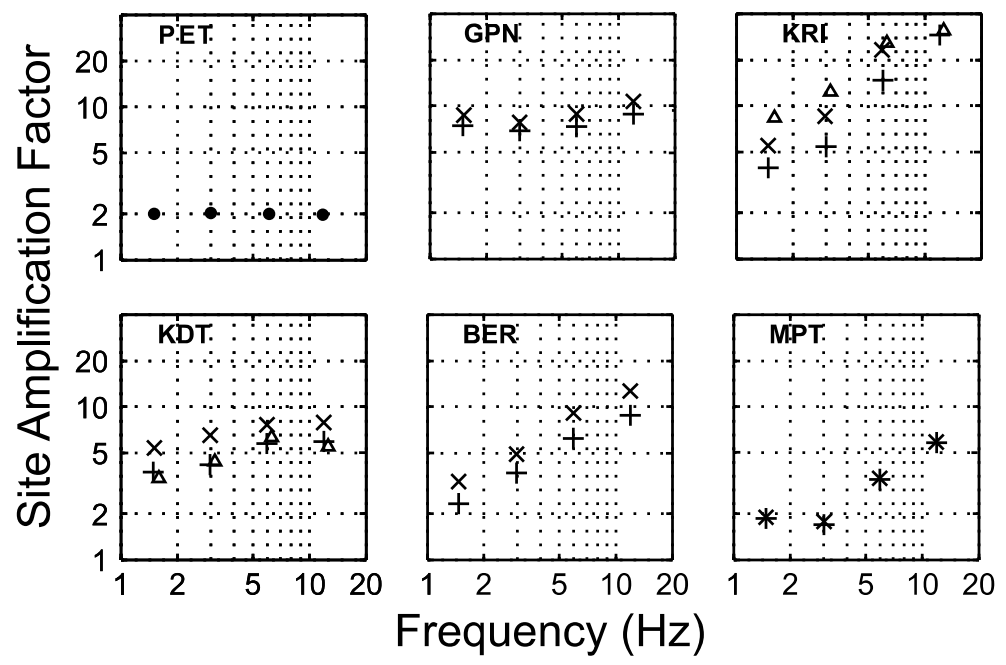

Fig. 6. Site amplification factors. Station PET was the reference station. Marks: $(+)$ results of inversion with Hybrid EnvGF; $(\times)$ same, with Theoretical EnvGF; $(\triangle)$ site correction factors estimated in an independent study after Petukhin et al. (2003).

\section{Results of Inversion}

Parameters of rupture. Table 3 shows best-fit values for rupture velocity $V_{R}$ and duration $T$ for each frequency band estimated by the grid search method within the following ranges: 2.0 to 4.0 for $V_{R}, 0.1$ to 10.0 for $T$. Values of the rupture velocity $V_{R}$ are the same for both types of EnvGF in the lower frequency ranges, and become different at higher frequencies. Actually, grid values show poor resolution for parameter $V_{R}$, probably due to the one-sided configuration of stations and/or small actual radiation area (see below): they are almost the same in a wide range $2.5-3.5 \mathrm{~km} / \mathrm{s}$. Therefore, in the following we freeze the common value $V_{R}=3.0 \mathrm{~km} / \mathrm{s}$ for all frequency bands and for inversions with both types of EnvGF.

Envelope fitting. Figure 4 shows examples of fitting observed envelopes with synthetic ones for two frequency bands near the maximum of the spectrum: $2-4$ and $4-8 \mathrm{~Hz}$, and for the case of Hybrid EnvGF. Except for one case (KDT, 2-4 Hz), the synthetic envelopes explain the observed ones quite acceptably. Inversion with the theoretical EnvGF produces similar results.

Spatial Distribution of Energy Radiation. Figure 5 shows the spatial distribution of HF energy radiation obtained for four frequency bands using hybrid and theoretical EnvGF's. In both types of inversions, the results are generally similar. For each frequency band, most of the energy (75-90 percent) was radiated from the second subfault, which includes the nucleation point of the rupture. Total energy (over 1-16 $\mathrm{Hz}$ band) is also similar in both cases: $W=1.6 \mathrm{e} 15 \mathrm{~J}$ and $1.2 \mathrm{e} 15 \mathrm{~J}$, respectively (that corresponds to $M_{S}=6.9$ and 6.85 based on Gutenberg-Richter relation: $\log W=$ $1.5 M_{S}+4.8$ (Gutenberg and Richter, 1956)). The difference can be regarded as a direction of further development of the HF radiator: the inversion with theoretical EnvGF indicates the eastward direction, whereas the inversion with the hybrid EnvGF envelope shows the opposite direction. The latter variant is in better agreement with the orientation of the aftershock cloud of the first-day aftershocks that were also spreading to the west (see Fig. 3).

Site Amplification Factor. Figure 6 shows inversion results for site amplification factor $S_{i}$ using hybrid and theoretical EnvGF $\left(S_{i}(\mathrm{PET}) \equiv 2.0\right.$, as explained above). For the stations KRI and KDT, independent estimates of spectral site amplifications were available (Petukhin et al., 2003). One can see that the inverted site factors match reasonably well (within a factor of 2) with independent estimates based on 
a much larger number of records. This suggests that other results of the inversion are also plausible.

Subfault duration parameter $T$. A large difference was observed in the results for the duration of energy radiation $T$ (see Table 3): estimated durations were much shorter for the inversion with the hybrid EnvGF (0.2 s) than for the inversion with theoretical EnvGF (2-4 s). It seems that this is the combined effect of the difference between the duration of the hybrid EnvGF and that of the theoretical EnvGF, source radiation directivity and quality of data and some other factors. The quality of the Kamchatkan strong-motion data is not sufficiently high to reliably resolve the time-dependent parameters. At this stage we cannot separate these effects. The hybrid envelope has a longer duration since it includes the effect of the pulse broadening due to diffraction. However, it smoothed out source radiation directivity effectively in averaging empirical envelopes. On the other hand, theoretical EnvGF does not correctly include the effect of the pulse broadening. Therefore, the actual value of parameter $T$ should be somewhat in the middle: $T \sim 1 \mathrm{sec}$, which is smaller than an estimation equal to the subfault size divided by rupture velocity: $10 / 3=3.3 \mathrm{sec}$. It means that the highfrequency radiation area may be smaller for this earthquake.

\section{Discussion and Conclusions}

The newly developed approach to envelope inversion using hybrid envelope Green's functions performed well, and showed some advantages with respect to the initial approach over theoretical Green's functions. The results of the new approach seem to be more reliable, and it can be recommended for future use for detailed studies. Its certain disadvantage is that it is needed to determine, beforehand, average empirical envelope shapes for the study area. For this reason, the basic method of Nakahara et al. (1998) can be still recommended for express analysis of source HF radiation.

The high-frequency source of the 1992 Avachinsky Gulf earthquake developed asymmetrically but not completely unilaterally, mostly in the western direction, at a velocity of about $3.0 \mathrm{~km} / \mathrm{s}$. Its length was about $30-40 \mathrm{~km}$. Accurate determination of the fault length was hampered by the fact that 75-90 percent of the total high-frequency energy was radiated immediately after nucleation, from the 5-10 km neighborhood of the nucleation point.

Acknowledgments. The study was initiated when A. G. was kindly invited to visit Tohoku University, Sendai, under the JSPS fellowship (Grant \#S97217). Kamchatka Experimental and Methodical Seismological Department (KEMSD GS RAS) kindly provided strong-motion data. The authors are grateful to Kojiro Irikura for encouragement for this work. Special thanks to Haruo Sato and Victor Pavlov provided constructive review comments on the manuscript. During this study, A. P. was partially supported by the
Postdoctoral Fellowship of the Japan Society for the Promotion of Science (Grant \#P98403).

\section{References}

Abubakirov, I. R. and A. A. Gusev, Estimation of scattering properties of lithosphere of Kamchatka based on Monte-Carlo simulation of record envelope of a near earthquake, Phys. Earth. Planet. Inter., 64, 52-67, 1990.

Gusev, A. A. and V. M. Pavlov, Deconvolution of squared velocity waveform as applied to the study of a noncoherent short period radiator in the earthquake source, Pure Appl. Geophys., 136, 235-244, 1991.

Gutenberg, B. and C. F. Richter, Magnitude and energy of earthquakes, Ann. Geofis., 9, 1-15, 1956.

Iida, M. and M. Hakuno, The difference in the complexities between the 1978 Miyagi-oki earthquake and the 1968 Tokachi-oki earthquake from a viewpoint of the short-period range, Natural Disaster Sci., 6, 1-26, 1984.

Kakehi, Y., K. Irikura, and M. Hoshiba, Estimation of high-frequency wave radiation areas on the fault plane of the 1995 Hyogo-ken Nanbu Earthquake by the envelope inversion of acceleration seismograms, J. Phys. Earth, 44, 505-517, 1996.

Nakahara, H., High-frequency envelope inversion analysis of the 2003 Tokachi-oki earthquake (Mw8.0). Abstracts, 2004 Japan Earth and Planetary Science Joint Meeting, CD-ROM, S052-P005, 2004.

Nakahara, H., T. Nishimura, H. Sato, and M. Ohtake, Seismogram envelope inversion for the spatial distribution of high-frequency energy radiation from the earthquake fault: Application to the 1994 far east off Sanriku earthquake, Japan, J. Geophys. Res., 103(B1), 855-867, 1998.

Nakahara, H., H. Sato, M. Ohtake, and T. Nishimura, Spatial distribution of high-frequency energy radiation on the fault of the 1995 Hyogo-ken Nanbu, Japan, earthquake (MW6.9) on the basis of the seismogram envelope inversion, Bull. Seismol. Soc. Am., 89, 22-35, 1999.

Nakahara, H. and R. Watanabe, Envelope inversion analysis of the 1999 Chi-Chi, Taiwan, earthquake for the spatial distribution of high-frequency seismic wave energy radiation on the fault plane, Eos Trans. AGU, 81(48), Fall Meet. Suppl., p. 881, 2000.

Petukhin, A. G., A. A. Gusev, E. M. Guseva, E. I. Gordeev, and V. N. Chebrov, Preliminary model for scaling of Fourier spectra of strong ground motion recorded on Kamchatka, Pure Appl. Geophys., 156, 445-468, 1999.

Petukhin, A. G. and A. A. Gusev, The duration-distance relationship and average envelope shapes of small Kamchatka earthquakes, Pure Appl. Geophys., 160, 9, 1717-1743, 2003.

Petukhin, A. G., A. A. Gusev, E. M. Guseva, E. I. Gordeev, and V. N. Chebrov, The first model for scaling of response spectra of strong ground motion recorded on Kamchatka, XXIII General Assembly of the International Union of Geodesy and Geophysics, Sapporo, Japan, 2003.

Sato, H., H. Nakahara, and M. Ohtake, Synthesis of scattered energy density for the non-spherical radiation from a point shear dislocation source based on the radiative transfer theory, Phys. Earth Planet Inter., 104, 113, 1997.

Saito, T., H. Sato, and M. Ohtake, Envelope broadening of spherically outgoing waves in three-dimensional random media having power-law spectra, J. Geophys. Res., 107, B5, 2002.

Zobin, V. M., E. I. Gordeev, V. I. Levina, V. F. Bakhtiarov, E. M. Guseva, D. V. Droznin, E. I. Ivanova, V. E. Levin, A. G. Petukhin, Yu. M. Khatkevich, and V. N. Chirkova, March 2, 1992, Earthquake (MLH=7.1) in the Gulf of Avacha, Kamchatka, and Associated Phenomena, Volc. Seis., 18, 687$702,1997$.

A. G. Petukhin (e-mail: anatolyp@geor.or.jp), H. Nakahara, and A. A. 